Please cite this article as: Krans, J, Pearson, D.G, Maier, B, Moulds, M.L, Contextual representations of negative images modulate intrusion frequency in an intrusion provocation paradigm, Journal of Behavior Therapy and Experimental Psychiatry (2015), doi: 10.1016/j.jbtep.2015.09.004.

\title{
CONTEXTUAL REPRESENTATIONS OF NEGATIVE IMAGES MODULATE INTRUSION FREQUENCY IN AN INTRUSION PROVOCATION PARADIGM
}

\author{
Krans, J., ${ }^{\mathrm{a}, \mathrm{c}}$, Pearson, D.G. ${ }^{\mathrm{b}}$, Maier, B ${ }^{\mathrm{a} 1}$, \& Moulds, M.L. ${ }^{\mathrm{a}}$ \\ ${ }^{\text {a }}$ School of Psychology, University of New South Wales, Sydney, Australia \\ ${ }^{\mathrm{b}}$ Department of Psychology, Anglia Ruskin University, Cambridge, UK.. \\ ${ }^{c}$ Clinical Psychology, University of Leuven, Leuven, Belgium
}

\section{Corresponding Author}

David Pearson

Department of Psychology

Anglia Ruskin University

East Road

Cambridge CB1 1PT, UK

Tel: +44 (0)8451962674

david.pearson@anglia.ac.uk

\footnotetext{
${ }^{1}$ Barbara Maier is now at the Goethe University in Frankfurt am Main
} 


\begin{abstract}
Background and objectives: To understand how memories of negative events become highly accessible in the context of trauma, we tested the hypothesis that contextual information modulates how easily intrusions can be provoked by perceptual stimuli.

Methods: Healthy participants viewed pictures depicting trauma scenes either with or without accompanying moderate (i.e. survival, recovery) or severe (i.e. fatality, permanent injury) outcome information. All participants viewed the same depictions of trauma scenes. Involuntary memories for the pictures were assessed using self-report diaries and an adapted version of the Impact of Event Scales (IES). A blurred picture perceptual priming paradigm was adapted to be used as an intrusion provocation task.

Results: The severe outcome group experienced a significantly higher frequency of intrusions on the intrusion provocation task in comparison to both moderate outcome and control (nocontext) conditions. The severe outcome condition did not increase intrusions on the selfreport diaries or the adapted IES. There was no effect of condition on ratings for the emotionality, self-relevance, valence, or seriousness of the trauma scenes.

Limitations: The analogue method should not be generalized directly to incidences of real-life trauma. It was unclear why differences in intrusion frequency were found in the provocation task only. The relative amount of individual conceptual and data-driven processing adopted by the participants was not assessed.

Conclusions: Manipulating contextual information that determines the meaning of sensoryperceptual features for a trauma scene can modulate subsequent intrusion frequency in response to visually similar cues.
\end{abstract}

KEYWORDS: intrusive memory; voluntary memory; perceptual priming; analogue trauma 
Perceptual and contextual information related to trauma events have been theorized to play unique roles in intrusion development (e.g., Brewin, 2014; Krans et al., 2013). For example, the importance of perceptual memory features in the cognitive model of PTSD proposed by Ehlers and Clark (2000). Intrusive trauma imagery is explained using a distinction between conceptual and data-driven processing in memory (Roediger, 1990). Data-driven processing has been defined as "encoding that focuses on the surface level (i.e. sensory details) of information rather than its meaning" (Ehlers, Michael, Chen, Payne, \& Shan, 2006, p. 318), while conceptual processing is defined as encoding that "reflect[s] meaning or conceptual elaboration" (Roediger, 1990). It has been argued that if an individual lacks conceptual processing and engages mainly in data-driven processing (i.e. they process the sensory impressions of an event rather than its meaning), then the resulting trauma memory will demonstrate strong perceptual priming effects for accompanying stimuli (Ehlers \& Clark, 2000). Other models which attribute the occurrence of involuntary intrusions predominantly to sensory-perceptual information include the dual-representation theory of PTSD (Brewin, Gregory, Lipton, \& Burgess, 2010) and the Self-Memory System model of autobiographical memory (Conway \& Pleydell-Pearce, 2000). Many previous analogue trauma studies of intrusive memory have adopted a theoretical framework based on the assumption that greater contextualisation of memories should result in fewer involuntary recollections (e.g. Bourne, Frasquilho, Roth, \& Holmes, 2010; Glazer, Mason, King, \& Brewin, 2013). These models consider the data-driven and conceptual processing as an intraindividual style that interacts with the nature of the trauma.

In the present study we are examining the effects of conceptual and perceptual information on the nature of the (analogue) trauma on the development of intrusive memories. Conceptual processing here can be thought of in terms of resulting in abstract 
contextual memory representations which code the meaning of an event separately from associated sensory-perceptual representations (Pearson, 2014). In the general non-clinical memory literature it has been common for the encoding of data-driven and conceptual information to be manipulated using differential presentation of pictorial and verbally-based stimuli (e.g., Weldon, Roediger, Beitel, \& Johnston, 1995; McDaniel, Robinson-Riegter, \& Einstein, 1998; Vandierendonck \& Rosseel, 2000), and such paradigms can be adapted to examine involuntary memory intrusions. Pearson, Ross and Webster (2012) presented participants with pictures depicting real-life images of violence with or without additional contextual information specifying the words "war" or "crime". Results showed presence of contextual information significantly increased the frequency of involuntary intrusions, but without any effect on reported vividness or emotionality. Krans, Langner, Reinecke, and Pearson (2013) presented participants with pictures depicting traumatic events either alone or accompanied by contextual statements (e.g. "This woman was asleep when a fire started in her kitchen. She is unconscious and is being carried out of her house by firemen"), and also found the presence of contextual information increased intrusion frequency. Overall these findings demonstrate that the contextual interpretation of sensory-perceptual elements of a scene during encoding can be integral to their subsequent occurrence as traumatic intrusions.

However, one limitation of previous contextual studies is that the contextual information accompanying trauma stimuli was consistent with either moderate or severe interpretations of the visual stimuli. For example, the contextual statements used by Krans et al. (2013) did not differentiate between severe and moderate outcomes, leaving the relative consequences of contextual information that differs in meaning unknown.

The present study aimed to address the research question of whether the accessibility of memory representations for trauma material is determined solely by encoded sensoryperceptual information, or whether there is also a contribution made by more abstract 
cognitive components that specify meaning. We adopted a paradigm in which the available perceptual information for trauma scenes was held constant across all conditions, while only information affecting the interpretation of the scenes was manipulated. The study expands on the methodology of previous studies by adapting a perceptual priming paradigm to provoke intrusions (Lang, Moulds, \& Holmes, 2009; Krans, Näring, Holmes, \& Becker, 2009), allowing the effect of contextual information on intrusion frequency to be examined. In Ehlers and Clark's (2000) model of PTSD, persistent intrusions are associated with strong perceptual priming effects, in which data-driven processing of a trauma event results in a reduced perceptual threshold for encoded stimuli. Involuntary intrusions are triggered when an individual encounters external cues in the environment that share sensory-perceptual characteristics with stimuli encoded during the original event. For example, in one case study patterns of sunlight on a lawn triggered intrusions of car headlights for the victim of a car accident (Ehlers \& Clark, 2000, p. 326). The experimental evidence for perceptual priming triggering intrusive memories is compelling, although it largely originates from analogue trauma studies (Ehlers, Michael, Chen, Payne, \& Shan, 2006). For example, Michael and Ehlers (2007) asked participants to complete a blurred object identification task, and found that perceptual priming of the objects was enhanced if they preceded a "traumatic" picture story rather than a neutral one. Similarly, Sündermann, Hauschildt, and Ehlers (2013) found that neutral objects were more strongly primed if they had been presented within the context of a trauma story rather than a neutral one.

The main aim of the current study was to experimentally compare the effects of providing severe or moderate outcome contextual information on involuntary memory for analogue trauma stimuli. Following previously established procedures (Pearson \& Sawyer, 2011; Pearson et al., 2012; Krans et al., 2013), we recruited healthy participants and presented them with pictures depicting negative emotional material. One group of 
participants received additional contextual information for each picture specifying a moderate outcome that placed emphasis on survival and recovery (e.g. "There were many survivors") while a second group received contextual information specifying a severe outcome for the same pictures which placed emphasis on fatality and permanent injury (e.g. "There were few survivors"). A third control group viewed the pictures alone without any accompanying contextual information. Because all participants viewed exactly the same pictures the surface sensory details available for encoding were held constant across the three groups, and only the encoding of meaning was manipulated; i.e. what participants believed the pictures were representing. As meaning was specified by the verbal textual descriptions rather than the sensory content of the pictures themselves, this manipulation affects the content of the available conceptual information rather than sensory information. We sought to examine whether there was a significant difference between the severe outcome and moderate outcome contextual conditions; particularly whether contextual information specifying a severe interpretation of scene content was associated with a significantly higher rate of involuntary intrusions.

\section{Method}

This study was approved by the Human Research Ethics Advisory Panel (Panel C, Behavioural) at the University of New South Wales (HREAP 123-162).

\section{Participants}

In total 120 participants were tested. Data was lost for one participant due to a technical error and seven participants failed to return for the second session. A further two participants reported depression levels in the moderate and severe range and were also excluded from the analysis. The final dataset contained data from 110 participants (moderate outcome context condition $n=37,26$ female; severe outcome context condition $n=38,17$ female; control condition $n=35,21$ female) including 64 women and 46 men with a mean 
age of $M=24.43, S D=5.16$, range 18 to 46 years old. Participants were 67 students of various courses and of various degrees (from undergraduate to $\mathrm{PhD}$ ) and 43 Sydney community participants. Psychology undergraduate participants received course credit for participation whereas other participants were reimbursed $\$ 30$ for their time. Exclusion criteria were: panic attacks, panic disorder (current and lifetime), PTSD (current and lifetime), major depressive episode (current and lifetime), psychotic episode (current and lifetime), blood phobia, and history of fainting.

\section{Materials}

Sample characteristics. Trait anxiety was measured with the State-Trait Anxiety Inventory-Trait version (STAI-T; Spielberger, Gorsuch, Lushene, Vagg, \& Jacobs, 1983). Levels of depression were measured with the Beck Depression Inventory-II (BDI-II; Beck, Steer, \& Brown, 1996). Negative mood state was measured using five single-item VAS scales on which participants rated current happiness, sadness, horror, anger, and disgust on a scale from 0 (not at all) to 10 (extremely) (Davies \& Clark, 1998). These scores were combined into a single negative mood score (happiness reversed).

Negative pictures. A selection of 18 negative pictures (595 x 795 pixels) was taken from a previous study in which negative pictures were used to induce intrusive images (Krans et al, 2013). Fourteen of these pictures were taken from the International Affective Picture System (IAPS; Lang, Bradley, \& Cuthbert, 1997) and four were selected from the internet (Krans et al., 2013). All pictures had a valence rating between 1.5 and 3.5 on a scale from 1 (most negative) to 9 (most positive) with arousal ratings of at least 4.79 on a scale from 1 (low arousal) to 9 (high arousal).

For each picture, severe and moderate contextual statements about the event depicted in the picture and the outcome of the event were created. The contextual information consisted of two sentences. The first sentence described the event in the picture and was the 
same for the severe and moderate vignettes (e.g., "This airplane malfunctioned and was forced to make an emergency landing"). The second sentence conveyed either a moderate outcome (e.g., "There were many survivors") or a severe outcome (e.g., "There were few survivors").

Task compliance and manipulation checks. Participants rated the extent to which they paid attention to the pictures, plus their emotionality, self-relevance, and seriousness on a 0 (none at all) to 10 (total attention/extremely emotional/self-relevant/serious) scale. Participants rated the valence of the pictures on a scale from -5 (very negative) to 5 (very positive).

Intrusive images. Three measures were used to assess the frequency of intrusive images of the negative pictures (Krans, Näring, Holmes, \& Becker, 2010). Participants recorded their intrusive images in a diary for seven days (e.g., Holmes, Brewin, \& Hennessy, 2004). Intrusive images were defined as images or memories of the pictures that popped into mind spontaneously and not when deliberately thought about. Participants were instructed to try and record their intrusive images as soon as they occurred and to check the diary at a fixed time each day for any omissions. For each entry the diary provided space to write a brief description of the content and ratings of the level of distress, vividness and detail associated with each intrusion on a scale from 0 (not at all) to 10 (extremely).

The intrusion provocation task (Lang, Moulds, \& Holmes, 2009; Krans et al., 2010) involved a perceptual priming paradigm adapted from previous studies in which participants view blurred versions of previously viewed trauma pictures and recorded any involuntary intrusions in response to the perceptual cues. The presentation of blurred pictures has been established in the prior literature as an effective measure of perceptual priming (e.g., Ehlers et al, 2006; Ehler et al, 2012; Michael \& Ehlers, 2007; Drummey \& Newcombe, 1995). Pictures were selected based on a pilot study with a different sample of 16 undergraduate students at 
the University of New South Wales. In the pilot study participants were presented with all 21 pictures from Krans et al.'s (2013) study in three blocks of 7 pictures each. In one block the pictures were presented with moderate contextual information, in another block the pictures were presented with severe contextual information, and in the third block the pictures were presented without further information. Before and after participants viewed the three blocks of pictures, they were presented with blurred versions of these pictures. The blur was accomplished by applying a Gaussian blur with a 100 px radius and a cubism filter with tile size 10 and saturation 2.5 using GIMP (version 2.6.11). For each blurred picture, participants indicated its content and rated its valence. The mean number of correct ratings of content for the blurred pictures was $2.05(\mathrm{SD}=3.02)$ before viewing the non-blurred versions, and 6.29 $(\mathrm{SD}=4.74)$ after. The mean valence ratings were $1.89(\mathrm{SD}=1.35)$ for moderate outcome, $0.97(\mathrm{SD}=1.16)$ for severe outcome, and $1.01(\mathrm{SD}=1.18)$ for control. The 8 selected blurred pictures were rated as more negative after participants had seen the non-blurred version during the pilot study, compared to before they had seen the non-blurred pictures. Similarly, the 8 selected blurred pictures were also recognized more often after the nonblurred picture presentation than before. The pilot study ensured that the blurred pictures triggered intrusions because they presented reminders of the original negative pictures, and not because they were intrinsically negative or salient. The final 18 pictures were selected because their valence ratings were the most positive with the moderate contextual information than with the severe contextual information.

During the intrusion provocation task itself participants were presented (in random order) with blurred versions of eight of the 18 negative pictures, for $4 \mathrm{sec}$ each. Each picture was presented once. After all eight pictures were presented participants monitored their thoughts for $2 \mathrm{~min}$ and pressed a button each time they had an intrusion of any of the negative pictures. A beep sound indicated to them that the 2 min were up. Participants were instructed 
to only press the button if a spontaneous image involuntarily came into mind rather than a voluntary retrieval, with the same definition of intrusiveness used as with the diary and IESintrusion subscale measures.

Finally, intrusion frequency and avoidance over the past seven days were measured with an adapted version of the Impact of Event Scale (IES; Horowitzet al., 1979). Items were rated on a 4 -point scale $(0=$ not at all, $1=$ rarely, $3=$ sometimes, $5=$ often $)$. The intrusion subscale includes 7 items and has good internal consistency (0.86) and test-retest reliability (0.89) (Sundin \& Horowitz, 2002). The avoidance subscale included 8 items with good internal consistency (0.82) and test-retest reliability (0.79) (Sundin \& Horowitz, 2002).

Recognition and recall. The rationale underlying the intrusion provocation task is that the blurred images perceptually prime intrusions because they match encoded memory representations for the original pictures (Ehlers et al, 2006; Michael \& Ehlers, 2007;

Drummey \& Newcombe, 1995). As a control it was therefore important to establish that there was no overall group difference in recognition memory for the pictures, as otherwise the blurred images would not reliably provoke intrusions if the pictures had not been encoded into memory in the first place. Recognition memory for the distressing pictures was measured with a 2 alternative forced-choice task (Krans et al., 2013). One of the two choices was always a picture that had been presented during the first experimental session. The other was novel picture with matched content (e.g., both showing a person on fire). The location of the original and novel picture (on the left or right half of the computer screen) was random as was the order of picture pairs. For each answer the participants indicated how sure they were on a scale from 0 (not at all sure) to 10 (absolutely sure).

Similarly, the validity of the main contextual manipulation assumes there are no group differences in whether participants read and encoded the different verbal contextual descriptions or not. To control for this recall memory for the context information presented 
with the pictures was assessed with a cued-recall memory task. Pictures from the first experimental session were presented on the computer screen one by one. Participants were then asked "What happened in this picture? Please write down as much as possible of what you remember." The cued-recall test was designed to check whether participants remembered the outcome of the events in the pictures in line with their experimental condition. Therefore, answers that reflected an outcome according to the experimental condition (severe, moderate) were counted as correct, even if participants remembered other details incorrectly.

Diary compliance and demand. Compliance was measured with a single item VAS scale. Participants were asked to rate the extent to which the following statement was true: "I have often been unable (or have forgotten) to record my intrusive images in the diary" on a scale from 0 (not at all true) to 10 (extremely true of me) (Davies \& Clark, 1998). Thus, lower scores indicated higher compliance.

Participants were asked with an open question what they thought the goal of the study was. Answers were coded by two independent raters into a dichotomous variable for mentions of manipulation of intrusion frequency (present or absent). Inter-rater agreement was acceptable with $K=0.71, p<001$. Disagreements were resolved with discussion. Further, participants were asked to indicate whether they thought that their experimental condition (that is, receiving relatively moderate or severe outcome information, or receiving no information) had increased, decreased, or not affected their intrusion frequency.

\section{Procedure $^{2}$}

Participants read and signed a consent form and were screened for exclusion criteria. Eligible participants completed a demographic questionnaire, followed by the STAI-T, BDIII and MoodQ. Participants were then randomly allocated to moderate outcome, severe

\footnotetext{
${ }^{2}$ A measure of over-general memory (SCEPT; Raes, Hermans, Williams, \& Eelen, 2007) was completed at baseline for a different purpose and is not reported here. Also for a different purpose, the recognition test consisted of three trials, during which each picture pair was presented once. Only responses from the first trial were included for analysis in the present paper.
} 
outcome, or control conditions and viewed the trauma scenes. Participants were not instructed to imagine they were victims or part of the observed scene. After presentation, participants were asked to rate their overall attention for the pictures and their valence, emotionality, personal relevance, and seriousness. Afterwards, participants received the diary along with the diary instructions. Participants returned to the lab exactly one week later. In this followup session, the provocation task was completed first, followed by the IES, the diary compliance rating, the recognition and cued-recall memory tasks, and demand questions. All participants were tested individually without the experimenter in the testing cubicle. All measures were presented using Inquisit software (version 4.0.2).

\section{Results}

\section{Statistical approach}

Data was checked for multivariate outliers using Mahalanobis distances (Tabachnick \& Fidell, 1996). None were found. Comparisons between experimental conditions were conducted using analysis of variance (ANOVA). In the event that a dependent variable was not normally distributed according to Levene's statistic, a negative binomial regression with robust covariance estimation was run to account for skewed distribution and possible univariate outliers. ANOVAs were used unless otherwise mentioned. All tests were twotailed with significance level of $\alpha=0.05$. Effect size Cohen's $f$ is reported for significant ANOVA tests. Table 1 shows the means and standard deviations for all variables within and across experimental conditions.

\section{Sample characteristics}

Participants in the three experimental conditions were comparable in age, $F(2,107)=$ $1.20, p=.306$, gender (i.e., male/female ratio), $\chi^{2}(2)=5.09, p=.078$, trait anxiety, $F(2,107)$ 
$=0.20, p=.823$, level of depression, $F(2,107)=1.19, p=.308$, and negative mood state at baseline, $F(2,107)=0.81, p=.447$.

\section{Manipulation check}

There was a significant difference in attention ratings for the pictures between the three experimental conditions, $F(2,107)=4.37, p=.015, f=0.29$. Pairwise comparisons showed that participants in the control condition reported significantly lower attention ratings compared to participants in the moderate and severe outcome conditions, $p=.048$ and $p=$ .005 , respectively. There was no significant difference in attention ratings between the moderate and severe outcome conditions, $p=.371$.

There was no significant difference between the three experimental conditions regarding the emotionality ratings for the pictures, $F(2,107)=1.67, p=.193$, self-relevance ratings, $F(2,107)=0.26, p=.974$, seriousness ratings, $F(2,107)=0.20, p=.823$, or valence ratings for the pictures, $F(2,107)=2.03, p=.136$.

\section{Intrusive images and intrusion provocation}

The intrusion frequencies according to the diary, provocation task and IES-intrusion subscale were significantly and positively correlated with each other: diary-provocation, $r=.24, p=$ .012 , diary-IES, $r=.50, p<.001$, and provocation-IES, $r=.27, p=.004$.

Correlations were also performed between the three outcome measures (diary, provocation task and IES-intrusion subscales) and the mean ratings for attention, valence, self-relevance, seriousness, and emotionality of the pictures. Only self-relevance was found to significantly correlate with intrusion frequency, and then only for the diary $(r=.22, p=$ 
$.022)$ and the IES-intrusion subscale $(\mathrm{r}=.21, \mathrm{p}=.03)$. The correlation between self-relevance and the provocation task was only marginal $(\mathrm{r}=.17, \mathrm{p}=.082)$.

There was no significant difference between the experimental conditions in the number of intrusive memories reported in the diary, $F(2,107)=0.10, p=.904$. There was a marginally significant effect of experimental condition on the average distress that accompanied the diary intrusions, $F(2,107)=3.06, p=.051, f=0.24$. Pairwise comparisons showed that average distress was higher in the control condition than in the moderate outcome condition, $p=.015$, but not different from the severe outcome condition, $p=.202$. There was no significant difference between the moderate and severe outcome conditions, $p=$ .224. There was no significant effect of experimental condition on the vividness of the intrusions in the diary, $F(2,107)=1.79, p=.173$, although according to pairwise comparisons the vividness ratings were marginally significantly higher in the control condition than in the moderate outcome condition, $p=.062$; all other $p>.318$. There was no significant main effect of condition on the level of detail that accompanied the diary intrusions, $F(2,107)=1.94, p=.149$, although according to pairwise comparisons the level of detail was marginally significantly higher in the control condition than in the moderate outcome condition, $p=.052$; all other $p>.259$.

Intrusion frequency (indexed by the IES) did not differ according to experimental condition, $F(2,107)=0.21, p=.814$. There was no main effect of condition for IES avoidance, $F(2,109)=0.34, p=.711$.

A negative binomial regression analysis showed that there was a significant effect of experimental condition on the frequency of reported intrusions in the provocation task, $F(2$, $107)=7.77, p=.001^{3}$. Pairwise comparisons showed that the provocation of intrusions for

\footnotetext{
${ }^{3}$ Because there was a marginal difference in the sex-ratio between groups a reviewer requested we perform additional analysis controlling for gender. An ANOVA showed no main effect of gender $(\mathrm{F}<1)$ or an interaction between gender and condition $(F<1)$. There remained a main effect of condition $(F(2,104)=5.91 ; p=.004)$, with
} 
the trauma slides was significantly higher in the severe outcome condition than in the moderate outcome condition, $p=.001$, and the control condition, $p=.015$. There was no significant difference between the moderate outcome and the control condition, $p=.184$.

\section{Recognition and cued-recall}

The purpose of the recognition test was to establish that the difference in frequency of intrusions on the provocation task could not be accounted for simply in terms of group differences in overall recognition memory for the pictures. In fact, only 57 participants (out of 110) made one or more mistakes on the recognition test. Overall, these participants were more confident about their correct answers than their incorrect answers, $F(1,54)=157.99, p$ $<.001, f=1.71$. A negative binomial regression analysis showed no significant effect of condition on recognition memory performance, $F(2,107)=1.18, p=.311$, establishing that the moderate outcome and no-context groups did not encode fewer pictures than the severe outcome group

There was also no effect of experimental condition on performance on the cued-recall task according to an ANOVA, $F(2,73)=0.18, p=.671$. This establishes that the moderate outcome and no-context groups did not remember less of the contextual verbal descriptions than the severe outcome group.

\section{Diary compliance and demand}

There was no significant difference between conditions on the diary compliance rating, $F(2,107)=0.10, p=.905$. Diary compliance was overall very good, $M=1.98, S D=$ 2.35 .

intrusions higher for the severe outcome condition in comparison to moderate outcome $(\mathrm{p}=.002)$ and control $(\mathrm{p}=.009)$. Moderate outcome and control conditions did not differ $(\mathrm{p}=.568)$. 
There was no significant difference between conditions in the number of participants who mentioned intrusion modulation as their perceived goal of the study, $F(2,107)=0.38, p$ $=.685$. Moreover, ANOVAs showed no significant effects of demand on intrusion frequency in the diary, $F(1,108)=0.05, p=.832$, the provocation task, $F(1,108)=0.15, p=.696$, or, according to a negative binomial regression, the IES-intrusion subscale, $F(1,108)=2.66, p=$ .106.

There was no difference in the number of intrusions between participants who expected their experimental condition to increase, decrease, or not affect their intrusion frequency for the diary, $F(2,107)=0.61, p=.548$, the provocation task, $F(2,107)=0.20, p$ $=.816$, or the IES-intrusion subscale, $F(2,107)=1.76, p=.178$.

\section{Discussion}

The main aim of the present study was to examine whether the presence of severe outcome contextual information was associated with a higher frequency of intrusive imagery in comparison to moderate outcome context and no-context conditions. This was driven by the rationale that severe contextual interpretations of trauma events can contribute to the modulation of intrusion frequency (Pearson, 2012; 2014).

We found the severe outcome group experienced significantly higher frequency of intrusions on the intrusion provocation task in comparison to moderate outcome and control (no-context) conditions. In contrast, there was no effect on either the diary or adapted IES measures. The main finding was therefore that participants who viewed images with severe context information about the outcome of the event showed a more accessible memory representation, as measured by an intrusion provocation task, than did participants who received moderate outcome information or no information about the outcome of the depicted event. There was no difference in memory accessibility between participants who received moderate context or no context information. One possible explanation is that participants did 
not encode images in the moderate context and no context control conditions as well as participants in the severe outcome condition. However, this does not seem to be the case, as participants in both context conditions indicated they allocated more attention than did participants in the control condition. The demand characteristics measures also revealed no significant differences between the conditions, and there was no evidence that the perceived valence of the pictures was affected by the positive and negative outcome manipulation. There was also no difference between either the recognition or cued recall control measures (i.e., voluntary memory).

Together, these findings suggest it is not the case that encoding more information per se increases memory accessibility. Rather, information needs to be perceived as specifying a high severity of outcome in order to increase the frequency of intrusions provoked by exposure to similar perceptual cues. Previous studies have found that self-relevance can be an important factor in influencing intrusion frequency. For example, Krans (2013) found that undergraduate participants reported more intrusive memories of a distressing film clip when they thought the victim was also a student. However, in the present study there was no significant effect of the contextual manipulation on self-relevance ratings. Pearson (2012) similarly reported no effect of contextual information on participant ratings for self-relevance or the seriousness of a distressing film that depicted road traffic accidents, despite the overall presence of a significant correlation between self-relevance and the total number of involuntary intrusions. The present study also found that self-relevance ratings were significantly correlated with intrusion frequency, but this was only for the diary and IES measures, not for the intrusion provocation task. Overall our findings imply that modulation of memory accessibility produced by severe outcome information may be separable from any effects of contextual information related to an increased perception of self-relevance. 
Importantly, our experimental manipulation also did not affect the number of intrusive memories as reported in the one-week diary, or as measured with the IES. While all three measures significantly and positively correlated (replicating a previous finding by Krans et al, 2010), the low nature of the correlations suggests they are not measuring a single construct. There are important methodological differences between the methods which need to be considered. In the IES participants are asked to retrospectively judge the frequency of intrusions across the previous seven-days. This measure therefore has the largest temporal distance between when intrusions are experienced and when they are reported. Because the diary measure is completed daily this temporal distance is reduced, but the practicalities of completing the diary mean frequency judgments are often still retrospective in nature. The intrusion provocation task in contrast is truly 'online' in the sense that intrusions are reported immediately that they occur, and this may make it a more sensitive and direct measure in comparison to measures that rely on retrospective judgments (e.g., Banaji \& Hardin, 1994; Barrett, 1997). Furthermore, it can be expected that the provocation task would be more likely to induce intrusions because participants are being presented with blurred versions of exactly the same pictures they saw during encoding. This means that the informational overlap between retrieval cues and memory representations will be considerably higher for the provocation task than the other measures. For example, while completing the seven-day diary participants may have experienced intrusions that were spontaneously triggered by encountering environmental cues which resemble the encoded pictures (for example, seeing a plane or fire engine). However, they are extremely unlikely to encounter cues which exactly overlap with the surface features of the pictures as occurs in the provocation task. If easy triggering of intrusions is a function of strong perceptual priming for encoded trauma stimuli (i.e. Ehlers \& Clark, 2000; Michael \& Ehlers, 2007), then it can be expected that frequency of intrusions should increase as the degree of perceptual similarity between environmental cues 
and encoded trauma stimuli increases. Parallels can be drawn with the wider memory literature where recognition-based paradigms reveal different results than cued-recall tests, largely due to the higher degree of informational overlap between cues and memory traces with recognition-based recall (e.g., Craik \& McDowd, 1987; Perlmutter, 1979).

It was not an objective of our study to test the prediction made by Ehlers and Clark (2000) that intrusion frequency is modulated by a shift in the relative degree of individuals' conceptual versus data-driven processing during encoding, and we do not regard our findings as being incompatible with the central claims made by their model. That is, whereas conceptual and data-driven processing as outlined in the cognitive models reflects intraindividual information processing, our manipulation can be seen as a manipulation of the features of the 'trauma' itself (i.e., its severity). Our results can be seen to complement and expand on previous studies such as those by Michael and Ehlers (2007) and Sündermann et al. (2013), who demonstrated enhanced perceptual priming for objects presented in the context of traumatic rather than neutral picture stories. However, while these studies also found a significant correlation between the strength of priming effect and frequency of selfreported intrusions for stimuli up to two to four weeks after presentation, they did not test whether blurred picture stimuli directly provoke intrusions, as should be expected if perceptual priming plays a functional role in triggering involuntary recollection. Our study has established that a laboratory-based priming paradigm is effective in directly provoking intrusive memories, and that the frequency of intrusions can be significantly modulated by experimentally manipulating the accompanying contextual information.

Some important caveats need to be considered. While a priming paradigm was adapted to measure the frequency of provoked intrusions for blurred stimuli, we did not measure the strength of perceptual priming itself using an identification task. We also did not use any self-report measures of data-driven processing, and these potentially could be 
incorporated in follow-up studies (e.g. the Data-driven Processing Scale (DDPS); Halligan, Clark \& Ehlers, 2002). However, we note that Sündermann et al. (2013) found that scores on the DDPS were not significantly correlated with the magnitude of enhanced perceptual priming for blurred stimuli, and they conclude that post-task self-reports of data-processing may be too imprecise to measure the implicit perceptual processes modulating priming effects and related intrusions. Nevertheless, it would be interesting to see how the availability and content of conceptual information and the availability of sensory information about the trauma influences the intra-individual relative data-driven versus conceptual processing. For the intrusion provocation task our participants were instructed only to report intrusions of previously presented pictures, but the lack of content data for the intrusions means that we cannot be $100 \%$ certain that intrusions did relate to the encoded slides rather than unrelated autobiographical memories. The "online" nature of the provocation task means that participants could not immediately describe their intrusions, but future studies could incorporate a post-task questionnaire to provide retrospective content data in order to help establish participants were following the task instructions correctly.

A further general limitation of our study is that our use of an analogue trauma paradigm with healthy participants may have failed to adequately simulate the effects of extreme stress on encoding that lead to PTSD for real events. It is also possible that the requirement to monitor intrusion frequency may lead to enhanced voluntary retrieval that poorly duplicate how intrusions occur outside of laboratory conditions (Holmes, 2003). Therefore any direct generalisation of our findings to experiences of actual trauma survivors should only be undertaken with extreme caution, and preferably not without further research focused specifically on clinical samples.

In short, our findings suggest that the frequency of intrusions for trauma scenes provoked during an adapted perceptual priming task can be modulated by manipulating the 
meaning attributed to the pictures, even while perceptual surface level features are held constant. We conclude that while intrusive mental images of trauma may derive from initial perceptual experiences, they need not solely reflect sensory-based depictions in perceptual memory. This suggests that factors modulating the provocation of involuntary memories in response to associated perceptual cues may be more complex than initially assumed. 


\section{Acknowledgements}

Julie Krans was funded by a Rubicon fellowship of the Netherlands Organisation for Scientific Research (NWO). This study also received some support from a NHMRC Project Grant (1004151) awarded to Michelle Moulds. 


\section{References}

American Psychiatric Association. (2013). Diagnostic and statistical manual of mental disorders (5th ed. TR). Washington, D.C: APA.

Banaji, M.R., \& Hardin, C. (1994). Affect and memory in retrospective reports. In N. Schwartz and S. Sudman (Eds.), Autobiographical Memory and the Validity of Retrospective Reports, pp. 71-86, Springer-Verlag.

Barquero, B., \& Logie, R.H. (1999). Imagery constraints on quantitative and qualitative aspects of mental synthesis. European Journal of Cognitive Psychology, 11, S. 315333.

Barrett, L.F. (1997). The relationships among momentary emotion experiences, personality descriptions, and retrospective ratings of emotion. Personality \& Social Psychology Bulletin, 23(i10), p1100.

Beck, A.T., Steer, R.A., Ball, R., \& Ranieri, W. (1996). Comparison of Beck Depression Inventories-1A and -II in psychiatric outpatients. Journal of Personality Assessment, 67, 588-597.

Beck, A.T., Steer, R.A., \& Brown, G.K. (1996). Manual for the Beck Depression InventoryII. San Antonio, TX: Psychological Corporation.

Berntsen, D. (1998). Voluntary and involuntary access to autobiographical memory. Memory, 6, 113-14

Berntsen, D. (2010). The unbidden past: involuntary autobiographical memories as a basic mode of remembering. Current Directions in Psychological Science, 19(3), 138-142.

Bourne, C., Frasquilho, F., Roth, A.D., \& Holmes, E.A. (2010). Is it mere distraction? Peritraumatic verbal tasks can increase analogue flashbacks but reduce voluntary memory 
performance. Journal of Behavior Therapy and Experimental Psychiatry, 41(3), 316324.

Brewin, C.R. (2014). Episodic memory, perceptual memory, and their interaction: Foundations for a theory of posttraumatic stress disorder. Psychological Bulletin, 140(1), 69-97.

Brewin, C.R., \& Burgess, N. (2014). Contextualisation in the revised dual representation theory of PTSD: A response to Pearson and colleagues. Journal of Behavior Therapy and Experimental Psychiatry, 45(1), 217-219.

Brewin C.R., Gregory J.D., Lipton M., Burgess N. (2010). Intrusive images in psychological disorders: Characteristics, neural mechanisms, and treatment implications. Psychological Review, 117, 210-232.

Chambers, D., \& Reisberg, D. (1985). Can mental images be ambiguous?Journal of Experimental Psychology: Human Perception \& Performance, 3, 317-328.

Conway, M. A. (2001). Sensory-perceptual episodic memory and its context: autobiographical memory. Philosophical Transactions of the Royal Society of London $B, 356,1375-1384$

Conway, M. A., \& Pleydell-Pearce, C. W. (2000). The construction of autobiographical memories in the Self-Memory System. Psychological Review, 107, 261-288.

Craik, F.I., \& McDowd, J.M. (1987). Age differences in recall and recognition. Journal of Experimental Psychology: Learning, Memory, and Cognition, 13(3), 474-479.

Davies, M., \& Clark, D. (1998). Predictors of analogue post-traumatic intrusive cognitions. Behavioural and Cognitive Psychotherapy, 26, 303-314.

Ehlers A., \& Clark D.M. (2000). A cognitive model of posttraumatic stress disorder Behaviour Research and Therapy, 38, 319-345. 
Ehlers, A., Hackmann, A., \& Michael, T. (2004). Intrusive reexperiencing in posttraumatic stress disorder: Phenomenology, theory, and therapy. Memory, 4, 403-415

Ehlers, A., Michael, T., Chen, Y. P., Payne, E., \& Shan, S. (2006). Enhanced perceptual priming for neutral stimuli in a traumatic context: a pathway to intrusive memories? Memory, 14, 316-328.

Ehring, T. \& Ehlers, A. (2011). Enhanced priming for trauma-related words predicts posttraumatic stress disorder. Journal of Abnormal Psychology, 120, 234-239.

GIMP GNU Image Manipulation Program 2.6.1 [Computer software]. (1995-2008). Kimball, S., Mattis, P., \& GIMP Development Team.

Glazer, D.A., Mason, O., King, J.A., Brewin, C.R. (2013). Contextual memory, psychosisproneness, and the experience of intrusive imagery. Cognition and Emotion, 27, 150157.

Hackmann, A. (2011). Imagery rescripting in posttraumatic stress disorder. Cognitive and Behavioral Practice, $18,424-432$.

Halligan, S.L., Clark, D.M., \& Ehlers, A. (2002). Cognitive processing, memory, and the development of PTSD symptoms: two experimental analogue studies. Journal of Behavior Therapy and Experimental Psychiatry, 33, 73-83.

Holmes, E.A. (2003). Intrusive, emotional mental imagery and trauma: Experimental and clinical cues. Imagination, Cognition and Personality, 23 (2-3), 147-154.

Holmes, E.A., Arntz, A., \& Smucker, M.R. (Eds.). (2007). Imagery rescripting in cognitive behaviour therapy: Images, treatment techniques and outcomes. [Special issue]. Journal of Behviour Therapy and Experimental Psychiatry, 38,297-305.

Holmes, E. A., \& Bourne, C. (2008). Inducing and modulating intrusive emotional memories: a review of the trauma film paradigm. Acta Psychologica, 127, 553566. 
Holmes, E.A., Brewin, C.R., \& Hennessy, R.G. (2004). Trauma films, information processing, and intrusive memory development. Journal of Experimental Psychology: General, 133, 3-22.

Holmes, E.A. \& Mathews, A. (2010). Mental imagery in emotion and emotional disorders. Clinical Psychology Review, 30, 349-362.

Horowitz, M., Wilner, N., \& Alvarez, W. (1979). Impact of Event Scale: A measure of subjective stress. Psychosomatic Medicine, 41, 209-218.

Hout, M.A. van den, Bartelski, N., \& Engelhard, I.M. (2012). On EMDR: eye movements during retrieval reduce subjective vividness and objective memory accessibility during future recall. Cognition and Emotion, 27, 177-184.

Hout, M.A. van den, Engelhard, I.M., Rijkeboer, M.M., Koekebakker, J., Hornsveld, H., Leer, A., Toffolo, M.B.J., \& Akse, N. (2011). EMDR: Eye movements superior to beeps in taxing working memory and reducing vividness of recollections. Behaviour Research and Therapy, 49, 92-98.

Hout, M.A. van den, Engelhard, I.M., Smeets, M., Hornsveld, H., Hoogeveen, E., Heer, E. de, Toffolo, M.B.J., \& Rijkeboer, M. (2010). Counting During Recall: Taxing of Working Memory and Reduced Vividness and Emotionality of Negative Memories. Applied Cognitive Psychology, 24, 1-9.

Inquisit 4.0.2 [Computer software]. (2012). Seattle, WA: Millisecond Software.

Kleim, B., Ehring, T., \& Ehlers, A. (2012). Perceptual processing advantages for traumarelated visual cues in post-traumatic stress disorder. Psychological Medicine, 42(1), 173-181.

Krans, J. (2013). The self and involuntary memory: Identifying with the victim increases memory accessibility for stressful events. Consciousness and Cognition, 22, 12981304. 
Krans, J., Langner, O., Reinecke, A., \& Pearson, D.G. (2013). Intrusive images and voluntary memory for affective pictures: Contextualization and dual-task interference. Journal of Behavioral Therapy and Experimental Psychiatry.

Krans, J., Näring, G., Becker, E.S., \& Holmes, E.A. (2009). Intrusive trauma memory: A review and functional analysis. Applied Cognitive Psychology, 23(8), 1076-1088.

Krans, J., Näring, G., Holmes, E.A., \& Becker, E.S. (2009). "I see what you're saying": Intrusive images from listening to a traumatic verbal report. Journal of Anxiety Disorders, 24, 134-140.

Lang, P.J., Bradley, M.M., \& Cuthbert, B.N. (1997). The International Affective Picture System. Gainesville, FL: NIMH Centre for the Study of Emotion and Attention, University of Florida.

Lang, T.J., Moulds, M.L., \& Holmes, E.A. (2009). Reducing depressive intrusions via a computerized cognitive bias modification of appraisals task: Developing a cognitive vaccine. Behaviour Research and Therapy, 47, 139-145.

Mace, J. H. (2004). Involuntary autobiographical memories are highly dependent on abstract cueing: the Proustian view is incorrect. Applied Cognitive Psychology, 18, 893-899.

Mace, J. H. (2005). Priming involuntary autobiographical memories. Memory, 13, 874-884.

Mace, J. H. (2006). Episodic remembering creates access to involuntary conscious memory: demonstrating involuntary recall on a voluntary recall task. Memory, 14(8), 917-924.

McDaniel, M.A., Robinson-Riegler, B., \& Einstein, G.O. (1998). Prospective remembering: Perceptually driven or conceptually driven processes? Memory \& Cognition, 26(1), $121-134$. 
Merckelbach, H., Dekkers, T., Wessel, I., \& Roefs, A. (2003). Amnesia, flashbacks, nightmares, and dissociation in aging concentration camp survivors. Behaviour Research and Therapy 41, 351-60.

Merckelbach, H., Muris, P., Horselenberg, R. \& Rassin,E. (1998). Traumatic intrusions as „worst case scenarios“. Behaviour Research \& Therapy, 36, 1075-1079.

Michael, T., \& Ehlers, A. (2007). Enhanced perceptual priming for neutral stimuli occurring in a traumatic context: two experimental investigations. Behavioral Research and Therapy, 45, 341-358.

Michael, T., Ehlers, A., Halligan, S. L., \& Clark, D. M. (2005). Unwanted memories of assault: What intrusion characteristics are associated with posttraumatic stress disorder? Behaviour Research and Therapy, 43, 613-628.

Pearson, D.G. (2014). Are C-reps contextual representations? A reply to Brewin and Burgess. Journal of Behavior Therapy and Experimental Psychiatry, 45(1), 220-222.

Pearson, D.G. (2012). Contextual representations increase analogue traumatic intrusions: Evidence against a dual-representation account of peri-traumatic processing. Journal of Behavior Therapy and Experimental Psychiatry, 43, 1026-1031.Pearson, D.G. (2014). 'Are C-reps Contextual Representations?: A Reply to Brewin and Burgess'. Journal of Behavior Therapy and Experimental Psychiatry, 45, 220-222.

Pearson, D.G., Deeprose, C., Wallace-Hadrill, S., Burnett Heyes, S. \& Holmes, E. (2013). Assessing mental imagery in clinical psychology: A review of imagery measures and a guiding framework. Clinical Psychology Review, 33, 1-23.

Pearson, D.G., Logie, R.H., \& Gilhooly, K.J. (1999). Verbal representations and spatial manipulation during mental synthesis. European Journal of Cognitive Psychology, 11, 295-314. 
Pearson, D.G., Ross, F.D.C., Webster, V.L. (2012).The importance of context: evidence that contextual representations increase intrusive memories. Journal of Behavior Therapy and Experimental Psychiatry, 43, 573-580.

Pearson, D. G., \& Sawyer, T. (2011). Effects of dual task interference on memory intrusions for affective images. International Journal of Cognitive Therapy, 4, $122-133$.

Perlmutter, M. (1979). Age differences in adults' free recall, cued recall, and recognition. Journal of Gerontology, 34(4), 533-539.

Pylyshyn, Z.W. (2006). Seeing and visualizing: It's not what you think. MIT Press, Cambridge, USA.

Raes, F., Hermans, D., Williams, J. M. G., \& Eelen, P. (2007). A sentence completion procedure as an alternative to the autobiographical memory test for assessing overgeneral memory in non-clinical populations. Memory, 15, 495-507.

Roediger, H. L. (1990). Implicit memory: retention without remembering. American Psychologist, $45,1043-1056$.

Spielberger, C.D., Gorsuch, R.L., Lushene, R., Vagg, P.R., \& Jacobs, G.A. (1983). Manual for the State-Trait Anxiety Inventory. Palo Alto, CA: Consulting Psychologists Press.

Sündermann, O., Hauschildt, M., \& Ehlers, A. (2013). Perceptual processing during trauma, priming and the development of intrusive memories. Journal of Behavior Therapy and Experimental Psychiatry, 44, 213-220.

Sundin, E.C., \& Horowitz, M.J. (2002). Impact of Event Scale: Psychometric properties. British Journal of Psychiatry, 180, 205-209.

Tabachnick, B.G., \& Fidell, L.S. (1996). Using multivariate statistics [3 ${ }^{\text {rd }}$ Ed.]. New York, NY: Harper Collins. 
Tsal, Y. \& Kolbert, L. (1985). Disambiguating ambiguous figures by selective attention. Quarterly Journal of Experimental Psychology, Section A: Human Experimental Psychology, 37, 25-37.

Vandierendonck, A., \& Rosseel, Y. (2000). Interaction of knowledge-driven and data-driven processing in category learning. European Journal of Cognitive Psychology, 12(1), $37-63$.

Weldon, M.S., Roediger, H.L., Beitel, D.A., \& Johnston, T.R. (1995). Perceptual and conceptual processes in implicit and explicit tests with picture fragment and word fragment cue. Journal of Memory and Language, 34(2), 268-285. 
Table 1. Means and standard deviations of all measures within and across experimental conditions.

\begin{tabular}{|c|c|c|c|c|c|c|c|c|c|}
\hline & & \multicolumn{2}{|c|}{$\begin{array}{l}\text { Moderate } \\
\text { outcome } \\
\text { condition } \\
(\mathrm{n}=37)\end{array}$} & \multicolumn{2}{|c|}{$\begin{array}{c}\text { Severe } \\
\text { outcome } \\
\text { condition } \\
(\mathrm{n}=38)\end{array}$} & \multicolumn{2}{|c|}{$\begin{array}{l}\text { Control } \\
\text { condition } \\
(\mathrm{n}=35)\end{array}$} & \multicolumn{2}{|c|}{$\begin{array}{c}\text { Total } \\
(\mathrm{n}=110)\end{array}$} \\
\hline & & $M$ & $S D$ & $M$ & $S D$ & $M$ & $S D$ & $M$ & $S D$ \\
\hline age & & 23.59 & 4.22 & 24.29 & 5.01 & 25.46 & 6.11 & 24.43 & 5.16 \\
\hline STAI-T & & 35.11 & 8.03 & 35.32 & 7.27 & 36.14 & 6.83 & 35.51 & 7.35 \\
\hline BDI-II & & 6.16 & 5.75 & 4.58 & 4.59 & 4.71 & 4.16 & 5.15 & 4.90 \\
\hline MoodQ & & 6.95 & 4.90 & 8.26 & 4.65 & 7.37 & 4.10 & 7.54 & 4.56 \\
\hline \multirow{5}{*}{$\begin{array}{l}\text { Picture } \\
\text { ratings }\end{array}$} & Attention & 8.86 & 1.65 & 9.16 & 1.20 & 8.20 & 1.35 & 8.75 & 1.45 \\
\hline & Emotionality & 6.24 & 2.56 & 7.21 & 2.46 & 6.49 & 2.08 & 6.65 & 2.40 \\
\hline & $\begin{array}{l}\text { Self- } \\
\text { relevance }\end{array}$ & 2.76 & 2.96 & 2.84 & 2.83 & 2.69 & 2.95 & 2.76 & 2.89 \\
\hline & Seriousness & 8.08 & 2.25 & 8.39 & 2.30 & 8.26 & 1.96 & 8.25 & 2.16 \\
\hline & Valence & -3.65 & 1.67 & -3.05 & 2.19 & -2.65 & 2.42 & -3.13 & 2.13 \\
\hline \multirow[t]{4}{*}{ Diary } & $\begin{array}{l}\text { Intrusion } \\
\text { frequency }\end{array}$ & 3.32 & 3.20 & 3.66 & 3.74 & 3.43 & 2.83 & 3.47 & 3.26 \\
\hline & Distress & 2.85 & 2.46 & 3.61 & 2.82 & 4.42 & 2.81 & 3.61 & 2.75 \\
\hline & Vividness & 3.44 & 2.96 & 4.05 & 2.85 & 4.74 & 2.93 & 4.07 & 2.93 \\
\hline & Detail & 3.30 & 2.89 & 3.87 & 2.85 & 4.64 & 2.95 & 3.92 & 2.20 \\
\hline $\begin{array}{l}\text { Provocation } \\
\text { task }\end{array}$ & & 3.27 & 2.74 & 7.16 & 6.66 & 4.20 & 3.22 & 4.91 & 4.86 \\
\hline \multirow[t]{2}{*}{ IES } & Intrusion & 5.73 & 5.91 & 6.47 & 5.74 & 5.80 & 4.87 & 6.01 & 5.50 \\
\hline & Avoidance & 7.03 & 7.41 & 8.13 & 8.45 & 8.54 & 8.35 & 7.89 & 8.03 \\
\hline $\begin{array}{l}\text { Recognition } \\
(\max =18)\end{array}$ & & 17.19 & 0.94 & 17.26 & 0.95 & 16.86 & 1.33 & 17.11 & 1.09 \\
\hline $\begin{array}{l}\text { Cued recall } \\
(\max =8)\end{array}$ & & 7.38 & 4.63 & 7.82 & 4.25 & - & - & 7.60 & 4.41 \\
\hline
\end{tabular}

